\title{
NMF on positron emission tomography
}

Bödvarsson, Bjarni; Hansen, Lars Kai; Svarer, Claus; Knudsen, Gitte

Published in:

IEEE International Conference on Acoustics, Speech and Signal Processing, 2007. ICASSP 2007.

Link to article, DOI:

10.1109/ICASSP.2007.366678

Publication date:

2007

Document Version

Publisher's PDF, also known as Version of record

Link back to DTU Orbit

Citation (APA):

Bödvarsson, B., Hansen, L. K., Svarer, C., \& Knudsen, G. (2007). NMF on positron emission tomography. In IEEE International Conference on Acoustics, Speech and Signal Processing, 2007. ICASSP 2007. CASSP. https://doi.org/10.1109/ICASSP.2007.366678

\section{General rights}

Copyright and moral rights for the publications made accessible in the public portal are retained by the authors and/or other copyright owners and it is a condition of accessing publications that users recognise and abide by the legal requirements associated with these rights.

- Users may download and print one copy of any publication from the public portal for the purpose of private study or research.

- You may not further distribute the material or use it for any profit-making activity or commercial gain

- You may freely distribute the URL identifying the publication in the public portal 


\section{NMF ON POSITRON EMISSION TOMOGRAPHY}

\author{
Bjarni Bodvarsson, Lars Kai Hansen \\ Informatics and Mathematical Modelling \\ Technical University of Denmark \\ DK-2800 Kgs. Lyngby, Denmark
}

\author{
Claus Svarer, Gitte Knudsen \\ Neurobiology Research Unit \\ Copenhagen University Hospital \\ DK-2100 Copenhagen, Denmark
}

\begin{abstract}
In positron emission tomography, kinetic modelling of brain tracer uptake, metabolism or binding requires knowledge of the cerebral input function. Traditionally, this is achieved with arterial blood sampling in the arm or as shown in [1] by non-invasive $\mathrm{K}$-means clustering. We propose another method to estimate time-activity curves (TAC) extracted directly from dynamic positron emission tomography (PET) scans by nonnegative matrix factorization (NMF). Since the scaling of the basis curves is lost in the NMF the estimated TAC is scaled by a vector $\alpha$ which is calculated from the NMF solution. The method is tested on a $\left[{ }^{18} \mathrm{~F}\right]$-Altanserin tracer ligand data set consisting of 5 healthy subjects. The results from using Kmeans clustering and NMF are compared to a sampled arterial TAC. The comparison is done by calculating the correlation with the arterial sampled TAC.
\end{abstract}

Index Terms - NMF, Positron emission tomography, Brain modeling

\section{INTRODUCTION}

Measurement of the cerebral input function in terms of the arterial plasma input TAC is required for assessment and validation of the quantification of receptor binding parameters on the basis of dynamic positron emission tomography (PET) images and kinetic models [1]. Alternative non-invasive methods for estimation of the input function have been described based on cluster analysis [1], based on linear decomposition methods such as independent component analysis (ICA) [2] [3], and by non-negative matrix factorization (NMF) of the signals in a region of interest [4] and on whole brain data [5]. The linear decomposition methods are preferred because they do not assume that voxels are dominated by the vascular signal, where the NMF method is attractive because of the relative straightforward estimation procedure. Furthermore, since the latter approach does not assume a specific form of the spatial distribution makes the NMF method attractive.

In this study the NMF method is used to extract the input TAC without the use of a set of predefined regions of interest and the results are subsequently compared to the results from
K-means clustering used in [1]. The estimated vascular TAC's are compared by correlation to the golden standard, namely the arterial sampling.

A challenge with the deconvolution methods as ICA and $\mathrm{NMF}$ is that the absolute scaling of the estimated basis curves is lost since the scaling can be applied to the mixing matrix and the basis function matrix without a change in the cost function. We propose a method of rescaling the TAC's by assuming that the mixing matrix must sum to one for each voxel. By performing this approximation the estimated vascular TAC from the NMF can be scaled so that it can be used directly in kinetic modelling without the use of an arterial sampling.

\section{METHODS}

$\mathrm{K}$-means and NMF are used to estimate a vascular signal from a PET-scan of the brain. These signals are then used as input curves instead of the sampled arterial curve. The correlations between the estimated and the sampled TAC's are calculated to compare the methods to one another.

\section{Non-negative matrix factorization}

The non-negative matrix factorization method (NMF) [6] performs a deconvolution of an observed data matrix. The data is represented as a linear combination of two factors. The factors can be positively combined only, no subtraction is allowed thus no cancellation of the components is possible. All elements in the data matrix and in the factors are positively constrained. This data structure is ideal for many observed signals in nature and in real life applications, since the data matrix can be a combination of different unknown signals that are mixed with each other in an unknown manner. The positive constraint is very useful when working with images, speech and many other applications where the original sources can be assumed to be positive and added together.

The NMF method describes the matrix $\mathbf{V}$, as a linear combination of the factors $\mathbf{W}$ and $\mathbf{H}$. All elements in $\mathbf{V}, \mathbf{W}$ and $\mathbf{H}$ are non-negative. 


$$
\mathbf{V} \approx \mathbf{W H}
$$

This can be applied to the problem at hand of extracting TACs; $\mathbf{V}$ being the dynamic PET image, $\mathbf{W}$ is the mixing matrix, and $\mathbf{H}$ contains the basis TACs. The Euclidian distance between the original data matrix $\mathbf{V}$ and $\mathbf{W H}$ is used as the cost function [6].

This method needs the number of sources, $\mathrm{K}$, to be predefined, no other parameters need to be set. To give the method the correct number of sources the knowledge must be a priori, or some analysis must be done to asses the number of components. In this case the NMF is done for a range of components, and the results are analyzed. Since both spatial and dynamic information is given in the solution, the solution can be validated. Overestimating the number of sources needed in a PET scan gives a result where the dynamics in the solution indicate unnatural behavior, or some of the spatial sources will be identical indicating that the original signals are split up, and thereby overfitting the data.

Since the data matrix $\mathbf{V}$ is approximated by the linear combination of the components in $\mathbf{W}$ and $\mathbf{H}$ the basis functions in $\mathbf{H}$ are not scaled correctly. Therefore a scaling procedure is required if the solution is to be used in kinetic modelling.

A method is proposed to calculate a vector $\alpha$ that will rescale $\mathbf{W}$ and $\mathbf{H}$ to the original scale. The vector $\alpha$ is elementwise multiplied with each row of $\mathbf{W}$, and the reciprocal transposed $\alpha$ is elementwise multiplied with each column of $\mathbf{H}$. Thereby the cost function will not change with this rescaling.

$$
\begin{aligned}
\mathbf{H}_{. i}^{\prime} & =\mathbf{H}_{. i} \cdot\left(\alpha^{T}\right)^{-1} \\
\mathbf{W}_{j .}^{\prime} & =\mathbf{W}_{j \cdot} \cdot \alpha \\
\mathbf{W}^{\prime} \mathbf{H}^{\prime} & =\mathbf{W H}
\end{aligned}
$$

where $\alpha=\left[\begin{array}{lll}\alpha_{1} & \ldots & \alpha_{K}\end{array}\right]^{T}$

Since $\mathbf{W}$ can be interpreted as the mixing matrix or as spatial images of the sources, a lemma can be defined for $\mathbf{W}$. In the mixing matrix each row must sum to $1 . \mathbf{W}$ is set to describe the mix of each source in each observation or pixel, and these mixing coefficients must sum to 1 . This means that if $\mathbf{W}$ consists of images of the estimated sources, then the summed image must equal 1 in each pixel.

$$
\mathbf{W}_{j .} \cdot \hat{\alpha}=1 \text {, for all rows } j
$$

If the above is assumed then $\hat{\alpha}$ is estimated from the NMF solution as:

$$
\hat{\alpha}=\left(\mathbf{W}^{T} \mathbf{W}\right)^{-1}\left(\sum_{j} \mathbf{W}_{j .}\right)^{T}
$$

\section{K-means clustering}

The K-means performs a clustering of the data into K components. Each voxel is assigned to one cluster only and the component is the mean of its voxels. Therefore the method has some limitations in which kind of data can be modelled. Since the estimated components is the mean of a group of voxels the estimated TAC's cannot have values smaller or larger than the mean value of the voxels. The advantage though is that the scaling is intact as opposed to the NMF method.

The K-means is computed as follows [7]:

1. Choose $\mathrm{K}$ cluster centers

2. Assign each voxel to its nearest cluster center

3. Recalculate the cluster centers so that the centers is the mean of its members

4. Repeat step 2 and 3 until the centers stabilize

The algorithm will converge to a local optimum so the algorithm can be repeated several times with different cluster center initializations to get the best solution.

\section{RESULTS}

\section{NMF results}

The NMF is performed on the five $\left[{ }^{18} F\right]$-altanserin PET-scans. The dynamic and spatial results are analyzed by visual inspection to find the appropriate number of sources. The main features to look for when analyzing the results, is to see if the TACs look natural. Does every TAC in the solution describe the current tracer behavior for a part of the brain, or is it simply overfitting the data. The TAC behavior can be validated by looking at the spatial distribution of each TAC.

\begin{tabular}{|c|c|c|c|c|c|}
\hline Subject & 1 & 2 & 3 & 4 & 5 \\
\hline $\mathrm{K}=2$ & 0.987 & 0.978 & 0.985 & 0.949 & 0.956 \\
\hline $\mathrm{K}=3$ & 0.968 & 0.968 & 0.970 & 0.967 & 0.969 \\
\hline $\mathrm{K}=4$ & 0.909 & 0.745 & 0.830 & 0.800 & 0.923 \\
\hline
\end{tabular}

Table 1. Correlations between NMF estimated and arterial sampled blood curve.

In figure 1 the 3 TACs found by the NMF are plotted for one subject. It can be seen that one of the TACs resembles a plasma TAC, with a clear peak at first and then a steady level until the end. Another TAC shows an increase with time which could indicate binding in the region of that source. The last source has the behavior of a non-binding region of the cerebral, as the TAC has a great increase at first and then a slow decrease, similar to the TAC of the cerebellum in which there is no binding [1]. 


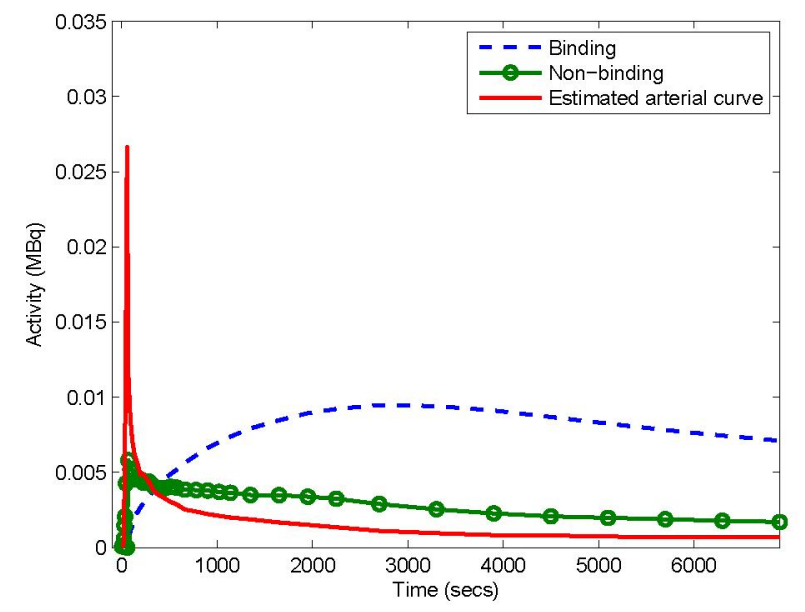

Fig. 1. NMF TAC's for subject 5 with $\mathrm{K}=3$

The correlation between the arterial sampled TAC and the $\mathrm{NMF}$ vascular component is used to evaluate the correctness of the factorization. The correlations can be seen in table 1, as seen for $\mathrm{K}=2$ and $\mathrm{K}=3$ the correlation is very high for all 5 subjects, above 0.94. For $\mathrm{K}=4$ the coefficient is lower for all subjects. This indicates that using 4 components is a overfitting of the data. To find out if 2 or 3 sources should be used to describe the PET data the spatial images of the NMF are investigated, and they show that the 3 components are independent and describe different regions of the brain. Therefore 3 components are used instead of 2 .

\section{K-means results}

The K-means clustering method is used to estimate the arterial input function which is then compared with the sampled arterial curve. In table 2 the correlation between the K-means estimated curve and the sampled arterial curve is repeated.

From the table it is seen that the correlations vary between subjects. Subject 1 has 0.63 as the highest and subject 5 has 0.96 as the highest correlation.

\begin{tabular}{|c|c|c|c|c|c|}
\hline Subject & 1 & 2 & 3 & 4 & 5 \\
\hline $\mathrm{K}=2$ & 0.47 & 0.70 & 0.84 & 0.92 & 0.55 \\
\hline $\mathrm{K}=3$ & 0.60 & 0.84 & 0.84 & 0.93 & 0.94 \\
\hline $\mathrm{K}=4$ & 0.61 & 0.84 & 0.82 & 0.93 & 0.94 \\
\hline $\mathrm{K}=5$ & 0.63 & 0.83 & 0.80 & 0.93 & 0.94 \\
\hline
\end{tabular}

Table 2. Correlations between K-means estimated and the arterial sampled blood curve.

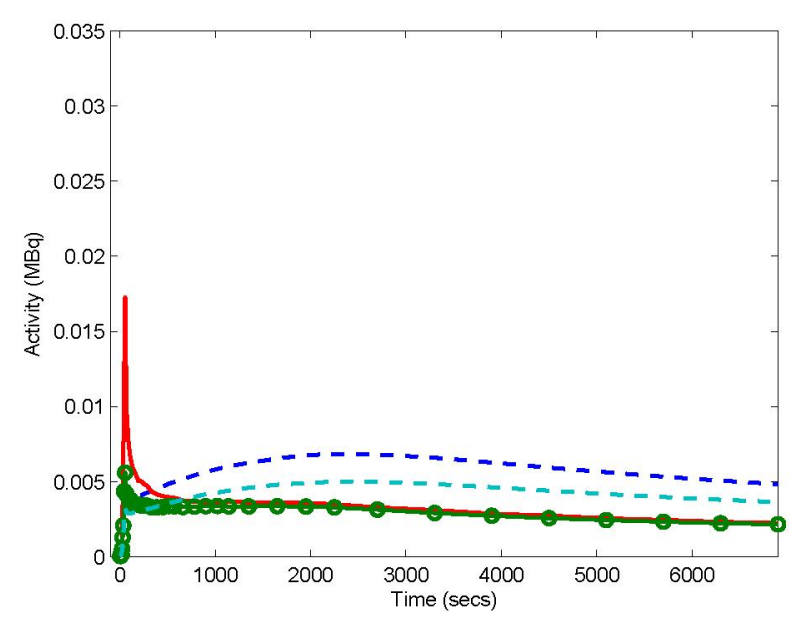

Fig. 2. K-means TAC's for subject 5 with $\mathrm{K}=4$

\section{DISCUSSION}

The spatial images of the $\mathrm{K}$-means and the $\mathrm{NMF}$ results are very similar, and the estimated vascular TAC can validated for both methods as the spatial component of the TAC is located in venous regions of the brain. The TAC's however need not be the same since the K-means TAC is a mean curve and does not account for the partial volume effect in the PET images. The arteries have diameters of around $2-6 \mathrm{~mm}$ and the maximum resolution in the images is $5-6 \mathrm{~mm}$. Therefore the arterial signal is not likely to be found as the only signal in any voxel. This is also seen when applying both the NMF and the K-means, since no pure arterial signal is found. The only vascular TAC found is the vein. The veins have larger diameters and are easier to identify in the PET scan.

When comparing the K-means and the NMF results it is seen that the NMF seems to be more stable, since the correlation between the NMF estimated TAC's and the arterial sampled TAC's are above 0.94 for all five subjects. In the case of using K-means the correlation varies between 0.61 and 0.94 $(\mathrm{K}=4)$. The amplitude in the NMF estimated TAC is much higher than when using the K-means method because of the partial volume effect.

Both methods give spatial information about the components. These can be manually validated to see if the solution is describing something meaningful or simply overfitting the data. Doing so for the NMF results it can be concluded that the 3 components describe different properties of the binding of the $\left[{ }^{18} \mathrm{~F}\right]$-Altanserin tracer in the brain. The 3 regions are vascular, specific binding and non-specific binding voxels. When looking at the $\mathrm{K}$-means results the same 3 regions can be identified, except that when using $K=4$, then there are two binding components with different levels which is also seen in figure 2. 
Both methods estimate a venous and not a arterial TAC which we are trying to find. This however is not a great problem since the venous signal from the brain is close to the arterial. The venous signal in the brain is different from that from the arm because of dispersion. So even though the estimated signal is venous like it can be used as an input TAC to the brain since it is close to the arterial TAC when using the NMF, with correlations of above 0.94 .

Since the NMF is able to cope with partial volume effect it could also be considered as a way to denoise the PET image simply by deducting the residuals from the NMF solution from the original PET data $\mathbf{V}$. Since the signals and the spatial components found make sense and look natural, the residuals in the the NMF solution could then be artifacts from the scan, and these can now be removed from the images. This however is a subject that needs further work.

\section{CONCLUSION}

It is shown that the NMF can be used to estimate the arterial input function. The method is superior to K-means clustering in this case because of the partial volume effects in the images. The issue of scaling the NMF components so that they can be used directly in kinetic modelling is overcome by assuming that the voxels sum to one in the mixing matrix.

\section{ACKNOWLEDGEMENTS}

The Center for Integrated Molecular Brain Imaging (Cimbi) is gratefully acknowledged for supporting this project.

\section{REFERENCES}

[1] Matthew Liptrot, Karen H. Adams, Lars Martiny, Lars H. Pinborg, Markus N. Lonsdale, Niels V. Olsen, Soren Holm, Claus Svarer, and Gitte M. Knudsen, "Cluster analysis in kinetic modelling of the brain: a noninvasive alternative to arterial sampling," Neurolmage, vol. 21, no. 2, pp. 483-493, 2004.

[2] Mika Naganawa, Yuichi Kimura, Tadashi Nariai, Kenji Ishii, Keiichi Oda, Yoshitsugu Manabe, Kunihiro Chihara, and Kiichi Ishiwata, "Omission of serial arterial blood sampling in neuroreceptor imaging with independent component analysis," NeuroImage, vol. 26, no. 3, pp. 885-890, 2005.

[3] Ji Young Ahn, Jae Sung Lee, Myoung Jin Jang, and Dong Soo Lee, "Noninvasive extraction of input function from carotid artery in $h_{2}^{15} o$ dynamic brain positron emission tomography using independent component analysis," Second International Workshop on Independent Component Analysis and Blind Signal Separation. Proceedings, pp. 493-7, 2000.
[4] Kyeong Min Kim, H Watabe, M Shidahara, Ji Young Ahn, Seungjin Choi, N Kudomi, K Hayashida, Y Miyake, and H Iida, "Noninvasive estimation of cerebral blood flow using image-derived carotid input function in $h_{2}^{15} \mathrm{o}$ dynamic pet," Nuclear Science Symposium Conference Record, 2001 IEEE, vol. 3, no. 1, pp. 1282-1285, 2001.

[5] Bjarni Bodvarsson and Martin Mørkebjerg, "Analysis of dynamic PET data," M.S. thesis, Informatics and Mathematical Modelling, Technical University of Denmark, DTU, Richard Petersens Plads, Building 321, DK-2800 Kgs. Lyngby, 2006, Supervised by Lars Kai Hansen, IMM.

[6] Daniel D. Lee and H. Sebastian Seung, "Algorithms for non-negative matrix factorization," in NIPS, 2000, pp. $556-562$.

[7] J. MacQueen, "Some methods for classification and analysis of multivariate observations.," In: Le Cam, L.M., Neyman, J. (Eds.), Proceedings of the Fifth Berkeley Symposium on Mathematical Statistics and Probability, vol. 1, no. 1, pp. 281-297, 1967. 